\title{
FSEE 2018 Preface
}

The 2018 International Seminar on Food Safety and Environmental Engineering (FSEE 2018) has been successfully held during Nov. 30 ${ }^{\text {th }}$-Dec. $2^{\text {nd }}, 2018$ in Guangzhou, China. FSEE is an international event organized by Guangdong Food and Drug Vocational College and coorganized by Guangdong Food Safety Quality Association, with the purpose to showcase advances in research and technologies in the fields of food safety and environmental engineering.

Through a variety of keynote lectures, oral and poster presentations and discussions, FSEE 2018 provides the medium for scientists and academicians from universities and industries all around the globe to promote and share various ideas and develop new collaborations in the scopes such as medical treatment and biomedical engineering, food safety and nutrition, food science and engineering as well as environmental science and environmental engineering.

This proceeding presents a selection from papers submitted to the conference from universities, research institute and industries. All of the papers were subjected to peer review by conference committee members. The papers accepted will be published by E3S Web of Conferences (ISSN:2267-1242), which will be submitted to EI Compendex, Scopus, Inspec, DOAJ, and CPCI (Web of Science) for indexing.

We would like to thank all the authors who have contributed to this volume and also to the organizing committee, reviewers, speakers, chairpersons and all the conference participants for their support to FSEE 2018.

The Committee of FSEE 2018 


\section{Organizing Committees}

\section{Conference Chair}

Prof. Yonghua Wang (Vice-president and doctoral supervisor of the school of Food Science and Engineering of South China University of Technology)

Prof. Yanyun Zheng (President of Guangdong Food and Drug Vocational College)

\section{Technical Program Committee}

Prof. Guoping Huang (Guangdong Food and Drug Vocational College)

Dr. R. Pandiselvam (ICAR-Central Plantation Crops Research Institute, India)

Dr.Mohammadali Ebrahimi-Nik (Ferdowsi University of Mashhad, Iran)

A. Prof. Jaroslaw Jakubowicz (Poznan University of Technology, Poland)

Associate Research. Jinliao He (Nanjin University)

Prof. Mohamed M H Mostafa (Central University of Technology, South Africa)

Prof. Yan Wu (Shandong University)

Prof. Samo Kralj (University of Maribor, Slovenia)

Dr. Goh Wan Inn (Department of Structural and Material Engineering, Malaysia)

Dr. Kandasamy Jayaraman (IIT Madras/Aerospace, India)

A. Prof. Haiyan Zhao (Qingdao Agricultural University)

A. Prof. Muhammad Rashed Al Mamun (Sylhet Agricultural University, Bangladesh)

Prof. Sumathy Krishnan (North Dakota State University, USA)

Dr. Hafiz Ansar Rasul Suleria (Deakin University, Australia)

A. Prof. Tao Zhang (China Agricultural University)

Prof. Changying Hu (Jinan University)

Prof. Junling Shi (Northwestern Polytechnical University)

A. Prof. Yongchao Huang (Guangzhou University)

Prof. Qiujin Zhu (Guizhou University)

Dr. Hui Li (Food Quality \& Safety Center, Nutrition \& Health Research Institute, COFCO Corporation)

A. Prof. Zhenbo Xu (South China University of Technology)

Prof. Kai Yan (Sun Yat-sen University)

Prof. Baojun $\mathrm{Xu}$ (Beijing Normal University-Hong Kong Baptist University United International College)

A. Prof. Quanle Xu (Northwest A\&F University)

Prof. Julius Mangisoni (Lilongwe University of Agriculture and Natural Resources, Malawi)

Prof. Donghao Li (Yanbian University)

Dr. Hongkang Zhang (Zhongkai University of Agriculture and Engineering)

Senior engineer. Zhi lan Peng (Zhoushan Institute for Food and Drug Control)

A. Prof. Yiwei Tang (Bohai University)

Yujie Wu (Guangxi Inspection and Quantiy) 